\title{
Thermodynamic Properties of Solids: Experiment and Modeling
}

Samrath L. Chaplot, Ranjan Mittal, and Narayani Choudhury

\section{1}

\section{Introduction}

While thinking of thermodynamic properties of solids, a wide variety of properties and phenomena come to mind. Perhaps the most notable are specific heat, phase transitions, thermal expansion, thermal conductivity, melting, and so on. The macroscopic thermodynamic properties [1-11] are determined by microscopic crystalline and electronic structure and atomic vibrations, and these are determined by the nature of bonding between the atoms. In this book, we focus on the understanding and modeling of these microscopic and macroscopic properties and the experimental techniques [12-21] used in their investigation.

The modeling of the structure, dynamics, and various thermodynamic properties is done either by the first-principles quantum mechanical methods [6, 7] or by the semiempirical methods [8-11] largely based on models of interatomic interactions. The former is computationally far more intensive; therefore, its application to complex structures has been more recent and somewhat limited because of the available computational resources. The latter has been more widely used. Both of these techniques are extensively covered in this book.

On the experimental side, a variety of microscopic and macroscopic techniques are in use. The visible light, infrared and X-ray photons, and thermal neutrons are most widely used microscopic probes. These spectroscopic techniques [12-17] generate a rich amount of complex data of all kinds of vibrational modes of various polarizations and symmetry. Theoretical lattice dynamical calculations [7-10] are necessary for optimal planning of the experiments and for the microscopic interpretation of complex experimental data. Macroscopic measurements of specific heat [18] and thermal expansion [19, 20] and use of high-pressure, high-temperature devices [21] are also particularly important for thermodynamic investigations. These experimental techniques and the interpretations of their results by theoretical techniques are presented in individual chapters. 
1.2

Spectroscopic Techniques and Semiempirical Theoretical Methods

The macroscopic thermodynamic properties are closely related to the microscopic dynamics of atoms. The collective vibrations of atoms in solids, which are called lattice vibrations, occur in discrete energies. These quanta of lattice vibrations are known as phonons.

Phonons are one of the fundamental excitations in a solid, and along with electrons they determine the thermodynamic properties of a material. In insulators and semiconductors, phonons play a prominent role. They directly contribute to a number of phenomena such as the thermal expansion, temperature dependence of mechanical properties, phase transitions, and phase diagrams. The understanding of phonon spectra, especially that of new materials, is essential for future technological developments.

The theoretical methods of lattice dynamics and the calculation of thermodynamic properties dealing with semiempirical and $a b$ initio approaches are covered in Chapters 3 and 8, respectively. The concept of phonons assumes that the atomic vibrations are harmonic in nature, which is strictly valid at low temperatures, typically below the Debye temperature of the solid. As a complementary tool, molecular dynamics simulation is especially useful in studying the dynamics at high temperatures and in understanding the mechanisms of solid-solid phase transitions and melting, and so on. The simulations are also discussed in Chapter 3.

Spectroscopic techniques aim to determine the characteristics of these phonons. There are three major spectroscopic techniques, namely, optical spectroscopy (Chapter 2), inelastic neutron scattering (Chapter 3), and inelastic X-ray scattering (Chapter 4), which are complementary to each other.

Recent developments in lasers, optics, and electronics have made a significant impact on the modern optical spectroscopic methods and instrumentations. The optical techniques of Raman, infrared, and Brillouin scattering are reviewed in Chapter 2. Here the authors present a detailed comparison among the three techniques and provide a theoretical and experimental methodology. The chapter also gives a detailed account of the contribution of optical spectroscopy methods for studying the vibrational properties of materials under extreme conditions of high pressures and high temperatures. The uses of the spectroscopic methods are illustrated by examples taken from recent literature.

Inelastic neutron scattering is a spectroscopic technique in which neutrons are used to probe the dynamics of atoms and molecules in solids. It is the main experimental technique for determining the phonon dispersion curves as it offers both energy and momentum well matched for studies of various materials. Chapter 3 describes the principles and recent applications of inelastic neutron scattering, theoretical lattice dynamics, and molecular dynamics simulations. The calculations provide microscopic insights into a variety of novel phenomena like high-pressure phonon softening, structural phase transitions, melting, and so on.

Inelastic neutron scattering experiments take long data acquisition time and require large sample volume. This situation has changed with the advances in 
synchrotron radiation instrumentation. The improvement in X-ray photon flux combined with advances in instrumentation capabilities has made it possible to carry out detailed phonon studies for small quantities of samples, which also enables measurements under very high pressure (Chapter 4). The X-ray beam can be focused down to micron size, which allows investigation of very small crystals. The technique of inelastic X-ray scattering has opened new and important opportunities in the investigation of dynamics of atoms.

\section{3}

\section{Thermal Measurement Techniques}

The specific heat of a material is one of the most important thermodynamic properties indicating its heat retention or loss ability. Specific heat measurements also reveal signatures of crystalline or magnetic phase transitions. The major factors contributing to the specific heat are the atomic vibrations and electrons, which are called the lattice and electronic specific heat, respectively. The relation of the specific heat to these important physical properties and phase transitions makes this subject especially interesting for experimental and theoretical investigations. Chapter 5 gives a detailed theory of specific heat and presents a number of theoretical models. The principles and experimental methods of calorimetry to measure heat capacity of solids are also described.

Thermal expansion, an important thermophysical property of materials, has been of considerable interest for research and development of technology. This is an important material property considered for any structural materials experiencing a temperature gradient. Examples range from metals and ceramic parts of cooking wares to highly sophisticated engineering mechanical structures, such as buildings, bridges, air/spacecraft bodies, vessels, kiln, furnaces, and so on. Thermal expansion data of ceramics have also been a prime consideration for the design of electrolytes and electrodes of solid oxide fuel cells as well as reactor technology.

The bulk thermal expansion is generally measured by techniques like dilatometer and interferometers, while lattice thermal expansion is generally determined by the diffraction methods like variable-temperature X-ray or neutron diffraction. The nature and type of interatomic bonding, polyhedra around the cations, and packing of atoms in the unit cell and so on are key features in governing the magnitude and anisotropy of thermal expansion behavior. The salient details of the diffraction method and its importance for thermal expansion measurements are discussed in Chapter 6.

\section{4}

\section{First-Principles Quantum Mechanical Methods}

The study of electronic structure in solids provides important insights into the atomic structure and electronic properties of materials. Chapter 7 describes the main 
theoretical approaches and computational techniques for electronic structure calculations. Various practical methods used to solve the electronic structure problem are discussed. Finally, the electronic structure and crystallographic phase transformations of elemental compounds under high external pressure are described by means of the first principles theory.

The $a b$ initio quantum mechanical computation methods have now become very useful research tool in the condensed matter physics. It is now possible to calculate a wide variety of material properties like electronic structure, elastic constants, phonon dispersion relations, and so on at high pressures and temperatures. Because of this, $a b$ initio methods are now quite useful in earth science since they provide data that are complementary to the experiments on material properties at extreme pressure and temperature conditions found in the earth's interior. The ab initio lattice dynamics is studied by means of a perturbative approach to the density functional theory (Chapter 8). The study of phonon dynamics in multilayers, surfaces, crystals with defects and impurities, and so on is very limited. Ab initio phonon calculation is expected to provide new insights into such systems.

\section{5}

\section{Outlook}

We have discussed above a variety of experimental and theoretical methods used in the study of thermodynamic properties and related topics. Two major developments seem to stand out in the immediate future. One is the availability of the next generation of neutron and synchrotron sources that would bring a revolution in the way experiments are carried out and the nature of information and knowledge that is derived. We may expect new physics about the local short-range structures and ordering in complex and mixed solids; real-time analysis of dynamical phenomena including phase transitions and growth of novel structures of low dimensions, especially nanostructures and multilayers; new applications in energy systems and environment and earth sciences [22]; and so on. The second major development is the spurt in massively parallel computing that would enable the modeling of the structures and dynamical phenomena just noted above in the context of nextgeneration experimental facilities. We hope and believe that the contents of various topics covered in this book would prove extremely valuable in these new developments.

\section{References}

1 Born, M. and Huang, K. (1954) Dynamical Theory of Crystal Lattices, Oxford University Press, London.

2 Venkataraman, G., Feldkamp, L., and Sahni, V.C. (1975) Dynamics of Perfect Crystals, MIT Press, Cambridge.
3 Bruesch, P. (1982) Phonons: Theory and Experiments I, Springer-Verlag, Berlin.

4 Bruesch, P. (1986) Phonons: Theory and Experiments II, Springer-Verlag, Berlin. 
5 Dove, M.T. (1993) Introduction to Lattice Dynamics, Cambridge University Press, Cambridge.

6 Martin, R.M. (2004) Electronic Structure: Basic Theory and Practical Methods, Cambridge University Press.

7 Baroni, S., de Gironcoli, S., Corso, A.D., and Giannozzi, P. (2001) Phonons and related crystal properties from density functional perturbation theory. Rev. Mod. Phys., 73, 515-562.

8 Chaplot, S.L., Choudhury, N., Ghose, S., Rao, M.N., Mittal, R., and Goel, P. (2002) Inelastic neutron scattering and lattice dynamics of minerals. Eur. J. Mineral, 14, 291-329.

9 Mittal, R., Chaplot, S.L., and Choudhury, N. (2006) Modeling of anomalous thermodynamic properties using lattice dynamics and inelastic neutron scattering. Prog. Mater. Sci., 51, 211-286.

10 Choudhury, N. and Chaplot, S.L. (2009) Inelastic neutron scattering and lattice dynamics: perspectives and challenges in mineral physics, in Neutron Applications in Earth, Energy and Environmental Sciences (eds L. Liang, H. Schober, and R. Rinaldi), Springer, New York, pp. 145-188.

11 Allen, M.P. and Tildesley, D.J. (1987) Computer Simulation of Liquids, Clarendon, Oxford.

12 Cardona, M. and Güntherodt, G. (eds) (1982) Topics in Applied Physics: Light Scattering in Solids II, Springer-Verlag.
13 Bacon, G.E. (1975) Neutron Diffraction, Oxford University Press, Oxford.

14 Dorner, B. (1982) Coherent Inelastic Neutron Scattering in Lattice Dynamics, Springer-Verlag, Berlin.

15 Price, D.L. and Skold, K. (1986) in Methods of Experimental Physics: Neutron Scattering Part A (eds K. Skold and D.L. Price), Academic Press, Orlando.

16 Burkel, E. (1991) Inelastic Scattering of XRays with Very High Energy Resolution, vol. 125, Springer Tracts in Modern Physics, Springer-Verlag, Berlin.

17 Krisch, M. and Sette, F. (2007) Inelastic Xray scattering from phonons, in Light Scattering in Solids, Novel Materials and Techniques, vol. 108, Topics in Applied Physics, Springer-Verlag, Berlin.

18 Sorai, M. (ed.) (2004) Comprehensive Handbook of Calorimetry and Thermal Analysis, John Wiley \& Sons, Ltd.

19 Yates, B. (1972) Thermal Expansion, Plenum Press, New York.

20 Krishnan, R.S., Srinivasan, R., and Devanarayann, S. (1979) Thermal Expansion of Crystal, Pergamon Press, Oxford.

21 Eremets, M. (1996) High Pressure Experimental Methods, Oxford Science Publications, Oxford.

22 Liang, L., Schober, H., and Rinaldi, R. (eds) (2009) Neutron Applications in Earth, Energy and Environmental Sciences, Springer, New York. 
